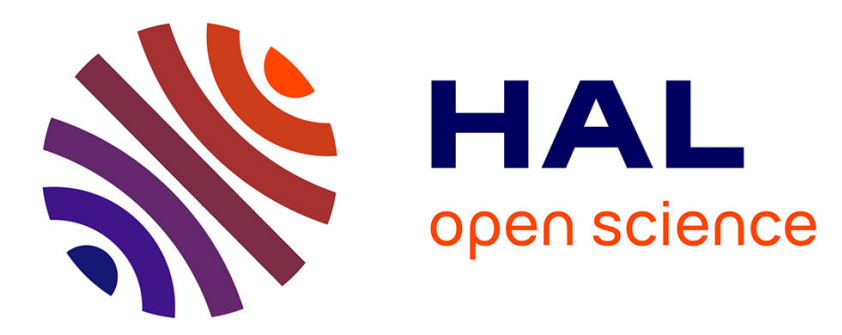

\title{
Immunogenicity of long-lasting recombinant factor VIII products
}

\author{
Mathieu Ing, Nimesh Gupta, Maud Teyssandier, Bernard Maillère, Marc \\ Pallardy, Sandrine Delignat, Sébastien Lacroix-Desmazes
}

\section{To cite this version:}

Mathieu Ing, Nimesh Gupta, Maud Teyssandier, Bernard Maillère, Marc Pallardy, et al.. Immunogenicity of long-lasting recombinant factor VIII products. Cellular Immunology, 2016, 301, pp.40-48. 10.1016/j.cellimm.2015.12.006 . hal-01292437

\section{HAL Id: hal-01292437 \\ https://hal.sorbonne-universite.fr/hal-01292437}

Submitted on 23 Mar 2016

HAL is a multi-disciplinary open access archive for the deposit and dissemination of scientific research documents, whether they are published or not. The documents may come from teaching and research institutions in France or abroad, or from public or private research centers.
L'archive ouverte pluridisciplinaire HAL, est destinée au dépôt et à la diffusion de documents scientifiques de niveau recherche, publiés ou non, émanant des établissements d'enseignement et de recherche français ou étrangers, des laboratoires publics ou privés. 


\section{Immunogenicity of long-lasting recombinant factor VIII products}

Mathieu Ing ${ }^{1,2,3}$, Nimesh Gupta ${ }^{4}$, Maud Teyssandier ${ }^{1,2,3}$, Bernard Maillère ${ }^{5}$, Marc Pallardy ${ }^{6}$, Sandrine Delignat ${ }^{1,2,3}$, Sébastien Lacroix-Desmazes ${ }^{1,2,3}$ on behalf of the ABIRISK consortium

${ }^{1}$ Sorbonne Universités, Université Pierre et Marie Curie, UMR_S 1138, Centre de Recherche des Cordeliers, F-75006, Paris, France; ${ }^{2}$ INSERM, UMR_S 1138, Centre de Recherche des Cordeliers, F-75006, Paris, France; ${ }^{3}$ Sorbonne Paris Cité, Université Paris Descartes, UMR_S 1138, Centre de Recherche des Cordeliers, F-75006, Paris, France; ${ }^{4}$ National Institute of Immunology, New Dehli, India; ${ }^{5}$ CEA-Saclay Institute of Biology and Technologies, Gif sur Yvette, France; ${ }^{6}$ INSERM, UMR996, Faculté Pharmacie, Université Paris Sud, France.

Corresponding author. Sébastien Lacroix-Desmazes, INSERM UMR_S 1138, Centre de Recherche des Cordeliers, Paris, F-75006 France - Tel: 0144278202 - Fax: 0144278194 sebastien.lacroix-desmazes@crc.jussieu.fr

Abbreviations. APC, antigen-presenting cells ; BcR, B cell receptor ; EMA, European Medicines Agency ; Fc, crystallizable fragment ; Fc $\gamma R$, receptor for IgG Fc portion ; FcRn, neonatal Fc receptor ; FVIIa, activated factor VII ; FVIII, Factor VIIII ; GLP-1, glucagon-like peptide 1 ; HA1, hemagglutinin ; HSA, human serum albumin ; IgG, immunoglobulin G ; IgM, immunoglobulin $\mathrm{M}$; LRP-1, lipoprotein receptor protein 1 ; MHC, major histocompatibility complex ; PEG, polyethylene glycol ; PTPs, previously treated patients ; PUPs, previously untreated patients ; Tregs, regulatory T cells ; VWF, von Willebrand factor

Abstract count: 158 words

Word count: 4842 
References count: 82 references 


\begin{abstract}
Replacement therapy for patients with hemophilia A using plasma-derived or recombinant factor VIII (FVIII) is complicated by the short half-life of the FVIII products and by the occurrence of neutralizing antibodies in a substantial number of patients. In the recent years, enormous efforts have been invested to develop new generations of coagulation factors with extended half-lives. Presumably, the use of long-lasting FVIII products should reduce the frequency of administration to the patients and drastically improve their quality of life. The question of their immunogenicity remains however unanswered as yet. The present review proposes a summary of the different strategies developed to enhance the half-life of FVIII, including fusion of FVIII to the Fc fragment of the human IgG1 or to human serum albumin, or attachment of polyethylene glycol. Based on the available literature, we hypothesize on the potential benefits or risks associated with each of the latter strategies in terms of immunogenicity of the newly derived hemostatic drugs.
\end{abstract}




\section{Introduction}

The past decades have witnessed extraordinary improvement in the treatment of bleeding disorders. Progressing from the Stone Age, with the use of whole blood and cryoprecipitate infusion, we have evolved to a modern time where recombinant coagulation factors are administered. Congenital disorders such as hemophilia A, that display life-threatening bleeding manifestations, have benefitted utmost from the development of new generation therapeutic factor VIII (FVIII), owing to the implication and commitment of the pharmaceutical field. Simultaneously, clinical studies as well as the implication of clinicians and basic researchers have promoted new treatment regimens (prophylaxis rather than "ondemand" treatment) that reduce the risks for hemarthrosis and arthropathy episodes and have improved the quality of life of hemophilic patients.

Despite the quality of FVIII in terms of efficacy and viral safety, the short half-life of FVIII imposes frequent administrations to provide an optimal protection of the patients from bleeding episodes. This is understandably associated with a limited adherence of the patients to prophylaxis regimen, thereby resulting in a lack of complete protection and higher treatment costs. Accordingly, the most recent developments for therapeutic coagulation factors have focused on extending their half-life in the blood: recombinant factors with longer residual time in circulation would cumulate the benefice of reducing the frequency of administration, thus improving the compliance of patients, and increasing the bleed-free timespan of the patients, thus reducing the risks for minor arthropathy-prone joint bleeds. In this context, several strategies are being exploited to optimize the pharmacokinetics of therapeutic FVIII, that include coupling of the effector protein to dimeric Fc fragments of human immunoglobulin G, to polyethylene glycol (PEG) or to human serum albumin (HSA).

The treatment of patients with hemophilia A using exogenous FVIII is complicated by the immunogenicity of the infused FVIII. Indeed, up to $30 \%$ of patients with severe hemophilia 
A, and up to $5 \%$ of patients with mild/moderate forms of the disease, develop anti-FVIII IgG antibodies following replacement therapy. The induced anti-FVIII IgG presumably affect the pharmacokinetics of the exogenously administered coagulation factors but, more critically, inhibit their pro-coagulant activity. The development of inhibitory anti-FVIII IgG, or 'FVIII inhibitors', represents a major clinical burden as well as a major societal concern owing to the additional costs that are associated with inhibitor management. To our knowledge, there is no available study that clearly establishes a correlation between the residual time of a molecule and its immunogenicity in humans. It is thus hazardous to predict the immunogenicity of long-acting FVIII products. Besides, FVIII exhibits a degree of immunogenicity that is unexpected given the fact that FVIII has no know pro-inflammatory role. In this review, we summarize the rationale for the different strategies developed to enhance the half-life of FVIII. Based on the available evidence, we further anticipate the consequences and limitations of coupling FVIII to 'half-life enhancers' for its immunogenicity in hemophilia A patients.

\section{Coupling therapeutic FVIII to human Fc fragments}

The interaction of the $\mathrm{Fc}$ domain of immunoglobulins with the neonatal $\mathrm{Fc}$ receptor $(\mathrm{FcRn})$ has been known for years as a physiological mechanism that protects IgGs from catabolism and confers them a long half-life in the blood [1]. Therefore, IgGs of the IgG1, IgG2 and IgG4 sub-classes are known to circulate in the body with a half-life of 3 weeks. The FcRn is expressed at many sites and by different cell types in mammals, where it mediates $\operatorname{IgG}$ transcytosis and IgG recycling in a $\mathrm{pH}$-dependent manner [1]. In particular, the $\mathrm{FcRn}$ expressed in the vascular endothelium participates in protection of $\operatorname{IgG}$ from degradation. Endothelial cells widely express FcRn and are able to internalize blood-borne molecules by pinocytosis. Under acidic $\mathrm{pH}$ in the early endosomes, the engulfed $\mathrm{IgG}$ binds the FcRn 
through their $\mathrm{C}_{\mathrm{H}} 2$ domain. Upon further acidification of the endosomes, IgG-FcRn complexes are redirected from the lysosomal degradation compartment to the cell surface. At neutral $\mathrm{pH}$, the bound $\mathrm{IgG}$ dissociates from FcRn and is released in the circulation. This property has been exploited to increase the residence time of coagulation factors in vivo. Besides, owing to the conformation of the Fc domain, Fc-fused proteins are generally endowed with improved biophysical characteristics in terms of stability and solubility.

In particular, a new generation of recombinant FVIII, referred to as Eloctate ${ }^{\mathrm{TM}}$ in North America, has been developed by fusing the dimeric $\mathrm{C}_{\mathrm{H}} 2-\mathrm{C}_{\mathrm{H}} 3$ domains of the human IgG1 Fc to the recombinant B domain-deleted FVIII. The post-translational modifications on the FVIII moiety are similar to that found on the commercially available B domain-deleted FVIII product, and the dimeric Fc carries two additional asparagine-glycosylated moieties. The addition of the Fc domain substantially increases the plasma half-life of FVIII and thus reduces the dosing frequency necessary to achieve prophylaxis in the patients. Thus, studies in pre-clinical models of hemophilia A have shown that fusion of the Fc to B domain-deleted FVIII confers a 2-fold increased half-life over recombinant FVIII, while preserving the specific activity of FVIII [2]. Indeed, the functionality of the Fc-fused FVIII in correcting whole blood clotting time has been demonstrated in FVIII-deficient mice, a model of severe hemophilia A, as well as in hemophilic dogs. The Fc-fused FVIII demonstrates a 1.5-fold extended half-life in human clinical trials as well as reduced annualized bleeding rates as compared to its non-fused counterpart [3-5].

From an immunogenicity standpoint, available data from clinical trials suggest that FVIII-Fc is a safe molecule since no inhibitor development has been reported under prophylaxis regimen $[5,6]$. It is important to note however that clinical trials have been performed only in previously treated patients (PTPs) with severe hemophilia A, who are at a lower risk of developing an alloimmune response to exogenous FVIII as compared to previously untreated 
patients (PUPs) [7]. Ongoing clinical trials performed in PUPs in Europe, which are required by the European Medicines Agency (EMA) prior to release of the drug on the European market, shall bring additional evidence in the coming years.

Presentation of Fc-fused FVIII as a magic drug devoid of immunogenicity needs to be confronted to the facts that therapeutic human monoclonal antibodies do induce neutralizing antibodies in a substantial number of patients [8] and the fused Fc domain has been used for enhancing the adjuvant properties of vaccine antigens $[9,10]$. Because the Fc portion of FVIII not only interacts with FcRn, but also with a number of receptors for the Fc portion of IgG (Fc $\gamma \mathrm{R})$, as well as receptors for Fc-linked glycans [11], there are little chances that the FVIIIFc fusion molecule will be immunologically inert. Yet, recent work by Krishnamoorty et al shows that the immune response to FVIII-Fc in FVIII-deficient mice, is less frequent, or less intense, than in the case of FVIII lacking the Fc fragment, when FVIII is injected at $50 \mathrm{IU} / \mathrm{kg}$ [12]. However, at higher concentrations, all FVIII products show the same degree of immunogenicity. The rest of this chapter is an attempt to anticipate the interactions of FVIIIFc with cells of the innate and adaptive immune system, and potential immunological consequences of infusing Fc-fused FVIII in patients.

Fc-mediated immune interactions. Navarrete et al. have previously observed that the marginal zone in the spleen is the primary site of FVIII accumulation in hemophilic mice [13]. Recently published data comparing the half-life and biodistribution of recombinant FVIII and FVIII-Fc intravenously administered to FVIII-deficient mice suggest that the accumulation of both molecules in the spleen does not show drastic differences [14]. The data also show that, as is the case for exogenous recombinant FVIII, the half-life and biodistribution of FVIII-Fc is largely dependent upon its interaction with endogenous von Willebrand factor (VWF). Interestingly, in view of the plethora of Fc receptors and Fc- 
binding cellular proteins expressed on immune cells, one may anticipate that FVIII-Fc is more prone to interact with immune cells than its native FVIII counterpart. In particular, one might foresee a synergistic effect of Fc-dependent and FVIII-dependent interactions with different types of endocytic receptors expressed by the same cells, although any repercussion in terms of immune activation or immune tolerance induction remains speculative.

Dendritic cells, monocytes, macrophages and neutrophils are the primary immune cells participating in the initiation of immune responses. Provided the constitutive expression of various Fc $\gamma$ receptors on these immune cells $[15,16]$, it is anticipated that FVIII-Fc will interact with the latter cell types via its Fc domain, in addition to accumulating at the site of vascular injury. In this context, we may expect a differential assessment of FVIII and FVIIIFc by the immune system, although predicting whether this will lead to increased or reduced immunogenicity is impossible. Dendritic cells are known to simultaneously express both the activating and inhibitory Fc $\gamma$ R. Presumably, FVIII-Fc-mediated signaling through the inhibitory FcyRIIB on dendritic cells should favor peripheral tolerance (Figure 1B), either by inhibiting dendritic cell activation or by limiting the processing of the exogenously acquired antigen [17]. However, the outcome of the immunological processing of FVIII-Fc will result from an equilibrium between the respective expression of the activating and inhibitory Fc $\gamma$ receptors, the affinity of the Fc portion for the different FcRs and for FcRn, and the concentration of FVIII-Fc in the circulation at a given time point. Of note, our unpublished data show that injection of high doses of C2 domain of FVIII fused to the mouse Fc $\gamma 1$ results in production of anti-C2 IgG by FVIII-deficient mice. Hence, the manifestation of immunogenicity may be highly dependent on the individual molecules that are fused to the Fc fragment.

Interestingly, Fc-mediated interactions also play a role in the context of secondary immune responses, once FVIII-specific $\mathrm{T}$ and $\mathrm{B}$ cells have been activated and have proliferated. 
Indeed, Fc $\gamma$ RIIB is the only Fc receptor that is constitutively expressed by B cells. It has been demonstrated that cross-linking of the B cell receptor $(\mathrm{BcR})$ and of the low affinity Fc $\gamma \mathrm{RIIB}$ inhibits $\mathrm{B}$ cell activation and proliferation in an antigen-specific manner, thus ablating humoral immune responses [18]. Despite the recent demonstration that Fc $\gamma$ RIIB is required for the differentiation of FVIII-specific memory B cells into antibody-secreting plasma cells in mice [19], the data suggest that FVIII-Fc may anergize FVIII-specific B cells and favor immune tolerance (Figure 1B).

Tolerance through regulatory $T$ cell epitopes. The $\mathrm{C}_{\mathrm{H}} 2$ domain of the $\mathrm{IgG} F \mathrm{Fc}$ fragment contains atypical $\mathrm{T}$ cell epitopes, which during co-presentation of $\mathrm{Fc}$ with associated antigen, has been shown to expand antigen-specific $\mathrm{CD} 4^{+} \mathrm{CD} 25^{\text {high }} \mathrm{Foxp} 3^{+}$regulatory $\mathrm{T}$ cells (Tregs) [20]. Fc-derived regulatory $\mathrm{T}$ cell epitopes, referred to as Tregitopes, were also found to confer tolerance to recombinant adeno-associated virus gene transfer vectors [21]. The presence of such Tregitopes in the FVIII-Fc molecules may present an advantage in favoring the induction of Tregs that would prevent the onset of deleterious anti-FVIII immune responses (Figure 1B). However, the efficiency of Tregitope-mediated Treg generation may depend on the frequency of administration of Fc-fused proteins, and variations in circulating concentrations of FVIII-Fc could drive opposite effects in terms of immune regulation.

The development of the FVIII-Fc platform nevertheless offers tremendous opportunities for generating less immunogenic FVIII products in the future. For instance, the introduction of mutations in the Fc portion may favor immune tolerance induction by fine-tuning the affinity for particular Fc receptors, provided that expanding the half-life of FVIII-Fc by raising the affinity of the Fc fragment for the FcRn does not alter the interactions with the inhibitory FcyRIIB receptor. In another example, the therapeutic infusion of FVIII-Fc via the subcutaneous route may open up new regimens for immune tolerance induction. Dendritic cells, and in particular skin dendritic cells, contribute to tolerogenic or immunogenic 
responses depending on the acquired activation signals [22]. Thus, the commitment of epidermal Langerhans cells in inducing antigen-specific tolerance could be exploited with FVIII-Fc [23].

Lastly, FcRn is expressed on the syncytiotrophoblast where it mediates the transfer of maternal IgGs to the fetal circulation [1]. In an animal model of mucopolysaccharidosis, Fcfused $\beta$-glucoronidase injected to pregnant mice was found to be transported through the placenta, thus correcting $\beta$-glucoronidase deficiency in the offspring [24]. In a recent proofof-concept study, the transplacental transfer of Fc-fused A2 and C2 domains of FVIII from pregnant mice to the hemophilic fetuses was able to confer long-lasting immune tolerance to therapeutic FVIII [25]. Tolerance to FVIII was mediated by FVIII-specific Tregs generated during the fetal development of the immune system. The latter work provides novel perspectives for the materno-fetal transfer of FVIII-Fc to to-be-born hemophilic babies, at the time of immune ontogeny, to confer life-long tolerance to FVIII.

\section{Coupling therapeutic FVIII to human albumin}

Accounting for $50 \%$ of total plasma proteins, albumin is the most prominent protein circulating in the blood. Serum albumin is a natural carrier for several endogenous hydrophobic molecules, such as fatty acids or thyroid hormones; it is also involved in scavenging free radical species that may be released in the blood. Albumin displays a long half-life of 20 days in vivo. This persistence is attributed to both a high hydrodynamic size, which prevents glomerular filtration by kidneys, and interactions with the FcRn receptor expressed on endothelial cells, which redirects the internalized albumin to the blood stream. Such an extended half-life makes serum albumin an attractive fusion partner to prolong the residence time of therapeutic drugs. Conjugation can be performed by a covalent attachment of albumin to the drug or by the fusion of the genes encoding human serum albumin and the 
therapeutic protein of interest [26].Several recombinant albumin-fused proteins have been successfully generated and are available on the market or currently tested in clinical trials [27]. For instance, albumin-coupled glucagon-like peptide 1 (Albiglutide®, GlaxoSmithKline) has been developed to treat type II diabetes. Clinical studies have demonstrated that a dramatic prolongation of circulation time after a single infusion of Albiglutide ${ }^{\circledR}$, from 5 minutes for the native glucagon-like peptide 1 (GLP-1) to 5 days for the albumin-fused molecule [28]. Recent phase 3 trial confirmed the efficacy of Albiglutide® [29], where Albiglutide ${ }^{\circledR}$ infusions successfully ameliorate glycemic control in type II diabetic patients. Nonetheless, $3.7 \%$ of treated patients were found to develop antiAlbiglutide ${ }^{\circledR}$ antibodies. The occurrence of anti-Albiglutide ${ }^{\circledR}$ antibodies was however transient and did not neutralize GLP-1 efficacy.

CSL-Behring® (Marburg, Germany) has developed an array of albumin-fused coagulation factors for the treatment of hemostatic conditions (patent WO 2011020866 A2, [30]). Inhibitor-positive patients with hemophilia A can be treated with activated factor VII (FVIIa). However, the short half-life of FVIIa (2.5 hours) requires chronic injections to maintain correct hemostasis. A recombinant FVIIa fused to albumin has been generated (rVIIa-FP) [31] that presents with a hemostatic activity similar to that of recombinant FVIIa. Clinical trials have highlighted the pharmacokinetic parameters of rVIIa-FP, with enhanced circulation time and lower clearance rate, and the safety of the product, as none of the rVIIa-FP-treated patients developed an anti-rVIIa-FP immune response [32]. Recombinant FIX-albumin, referred as rIX-FP, has also been produced and exhibits a 2- to 4-fold prolonged half-life compared to the non-fused form when administered to previously treated patients with severe hemophilic B [33]. Likewise, none of the participants developed anti-rIX-FP inhibitory antibodies. An albumin-fused FVIII has also been generated and patented (termed as rVIIIFP). However, rVIII-FP failed to maintain FVIII pro-coagulant activity and no further study 
was published [34]. Some studies have highlighted limitations for albumin conjugation [35], such as the risks of incorrect folding of chimeric proteins that is likely to lead to a loss of therapeutic activity.

Since albumin is the most abundant protein in the blood, it is not likely that exogenously administered albumin will be recognized as a foreign antigen. This is indirectly suggested by the fact that the massive intravenous administration of albumin to patients suffering from acute hypovolemia has never been reported to elicit anti-albumin antibodies [36]. Hence, albumin-fused coagulation factors are not expected to exhibit immunogenic properties, and should elicit immune tolerance or ignorance. Besides, albumin might mask by steric hindrance putative antigenic epitopes expressed on therapeutics and impede any deleterious recognition by immune cells that may lead to premature clearance or immunogenic uptake by antigen presenting cells (APC). Importantly, in silico $\mathrm{T}$ cell epitope mapping studies have identified six potential Tregitopes within human albumin [37]. One of the Tregitopes is also

found in bovine serum albumin. Interestingly, infusion of BSA in a murine model of atherosclerosis was shown to lower the severity of the pathology [38]. This was correlated with the expansion of circulating Tregs in vivo and production of immunosuppressive cytokines by splenocytes in vitro. However, a direct role for the Tregitope in the induction of tolerance was not demonstrated.

\section{PEGylation of therapeutic FVIII}

PEGylation consists in a covalent conjugation of polymers of ethylene glycol (PEG) to a carrier. PEGylation was found to extend the residence time of derivatized proteins $[39,40]$, and was therefore considered as an efficient strategy to improve drug delivery and has been applied to enhance the lifespan of a large number of drugs over the last decades. PEG conjugation affects the physicochemical features of the target protein, without altering its 
structure. In particular, the hydrophilic property of PEG increases protein solubility in the bloodstream and dramatically enhances the hydrodynamic size of the conjugate, thus tempering glomerular filtration rate and significantly prolonging the circulation time in the body [41]. Moreover, PEG prevents the aggregation of derivatized proteins and hampers interactions with catabolic receptors, which also impedes a premature clearance from the circulation.

Based on these findings, PEGylation has been extensively exploited to generate long-lasting recombinant coagulation FVIII, such as BAY 94-9027, BAX 855 and N8-GP. PEGylation of FVIII was shown to confer prolonged efficacy in hemophilic mice, as compared to unmodified FVIII. BAY 94-9027, a single branched 60 kDa PEG-coupled B domain-deleted FVIII developed by Bayer, not only retains a full activity after PEGylation, but also exhibited enhanced pharmacokinetic parameters, with a 2 to 3-fold increase of circulating half-life as compared to non-PEGylated FVIII [42]. Recent phase I clinical trial has confirmed the longer half-life in humans, although the extension was less significant than in animal models, with a 1.5-fold increase (18.5 hours versus 13 hours for its non-PEGylated version) [43]. Furthermore, infusion of BAY 94-9027 to patients was not reported to elicit inhibiting antibodies. Likewise, PEGylation of Baxter's recombinant full-length FVIII Advate ${ }^{\mathrm{TM}}$ (BAX 855, $20 \mathrm{kDa}$ PEG) has generated an improved molecule that exhibits a 1.8-fold extension of circulating time in primates compared to native FVIII and that delays by 3 folds the time to reach trough level [44]. PEGylation of FVIII impedes the binding of BAX 855 to the low density lipoprotein receptor protein 1 (LRP-1), a catabolic receptor for FVIII, which accounts, at least in part, for the longer persistence of the molecule in the bloodstream. A recent clinical trial has highlighted a modest 1.5-fold increase of BAX 855 half-life [45]. Prophylactic regimen performed over several months on PTPs was not associated with the generation of neutralizing antibodies. Similarly, Novo-Nordisk has developed a glycoPEGylated version of 
a B-domain deleted FVIII (N8-GP, 40 kDa PEG coupled to B domain) which displays a 2fold prolonged half-life in animal models of hemophilia A, as well as an extended hemostatic potential compared to uncoupled FVIII $[46,47]$. N8-GP was also shown to interact less with the catabolic receptor LRP-1. Clinical trials in PTPs with severe hemophilia A did not report the generation of anti-FVIII neutralizing antibodies [48].

From an immunological point of view, PEG theoretically gathers several characteristics that make it an ideal partner. PEGylation has been shown to annihilate the immunogenicity of heterologous proteins after administration to animals, as compared to the non-PEGylated counterparts [49]. The PEG moiety can mask structures that are recognized by endocytic receptors expressed on APC and hampers the uptake of the drug (Figure 1C). Besides, even if PEGylated biopharmaceutics are internalized, the processing that leads to the presentation of derived peptides onto major histocompatibility complex (MHC) class II molecules may be hampered by PEG-mediated steric hindrance. Alternatively, PEGylated peptides can be presented on APC surface, but may however not be recognized by $\mathrm{T}$ helper cells, thus preventing $\mathrm{T}$ cell activation, limiting the activation of antigen-specific $\mathrm{B}$ cells and, as a result, reducing the risk for the generation of anti-drug antibodies. In the case of FVIII, administration of BAY 94-9027 to hemophilic mice was correlated with a lower incidence of anti-FVIII antibodies and a reduction in the FVIII-specific T cell immune response. Besides, PEGylation, upon masking essential glycoprotein moieties involved in FVIII recognition, was shown to interfere with its uptake and presentation by human dendritic cells [50], and thus hindered the ensuing immunogenic presentation of FVIII-derived peptides to specific $\mathrm{CD} 4^{+} \mathrm{T}$ cells. Similarly, in vitro assays show that N8-GP binds less to human dendritic cells and is less internalized by these cells [46]. While any structural alteration of the FVIII molecule introduces a risk to enhance its immunogenicity, the generation of immunogenic B or T cell 
neo-epitopes within PEGylated FVIII is not supported by the available clinical studies, and no clear increase in the incidence of FVIII inhibitor has been reported up to date.

Safety and immunological aspect of PEG. Since PEG is a chemical compound that cannot be readily metabolized by the organism, concerns have been raised regarding its elimination/accumulation in the organism. It has been documented that PEG is cleared from the circulation through urinary excretion [51]. However, several studies have emphasized histological alterations in various organs and tissues, consecutive to the administration of PEG or PEGylated proteins. Infusion of PEGylated proteins was associated with a massive cytoplasmic vacuolation, evidenced in the kidney tubular epithelium [52] as well as in the reticuloendothelial system. These changes supposedly resulted from a deformation of lysosomes after the uptake of PEGylated proteins during their clearance process or by pinocytosis. Besides, the occurrence of foamy macrophages has been described after an iterative administration of PEG or PEGylated biotherapeutics [52-54]. PEG injection in rats led to a cellular vacuolation that occurred in different locations, such as lung alveolar macrophages, hepatic Kupffer cells or splenic subcapsular red pulp histiocytes, where PEG was found to accumulate [53]. Since the reticuloendothelial system is a component involved in the clearance of cellular debris and foreign bodies, accumulation of PEG and vacuolation at the level of macrophages are part of a physiological mechanism. Nevertheless, issues have been raised about a putative toxicity of PEG, especially in a context (i.e., hemophilia) where administration of the PEGylated drug is chronic and starts during infancy. Of note, administration of high doses of PEGylated proteins to rodents was associated with splenic hypertrophy and an extensive vacuolation of splenic histocytes [55]. Likewise, Young et al. demonstrated that infusion of maleimide-PEG-modified hemoglobin in primates is associated with the occurrence of foamy macrophages in the spleen and in the bone marrow [56]. Intriguingly, macrophage functions and phenotype were either not evaluated or not altered by 
vacuolation in vivo. However, mouse and human macrophages incubated with certolizumab pegol (PEGylated anti-tumor necrosis factor alpha, Cimziaß) in vitro showed a lower phagocytic ability for bacteria and fungi (report EMEA/664021/2009). Yet, no evidence regarding altered immune responses was found in vivo and the safety of Cimzia ${ }^{\circledR}$ was supported by preclinical and clinical studies, as no major adverse events was reported. Importantly, although vacuolation in tissues or in macrophages has been reported for several biopharmaceuticals commercially available or in clinical trials [51], the administration of BAX 855 to animals did not generate overt vacuolation [57].

While PEG is assumed to be neither antigenic nor immunogenic, an increasing number of studies provides evidence that administration of PEGylated products triggers unexpected antiPEG antibody production [58]. Thirty years ago, Richter and Akerblom demonstrated the generation of anti-PEG IgM antibodies in rodents after injections of PEGylated ovalbumin, while PEG injected alone did not elicit an antibody response $[59,60]$. In humans, anti-PEG antibodies have been reported in hepatitis C patients treated with PEGylated interferon, but the presence of anti-PEG antibodies was not associated with an impairment of the efficacy of interferon [61]. Conversely, in acute lymphoblastic leukemia, one third of the patients treated with PEGylated asparaginase presented with anti-PEG IgM antibodies, which were closely associated with a loss of function and an accelerated clearance of the molecule [62]. Whereas the exact mechanism for anti-PEG antibody elicitation remains unknown, several investigations have been performed to decipher the immunogenicity of PEG. Chronic intravenous infusion in mice of PEGylated liposomes, which are often used as a carrier for biopharmaceuticals, was correlated to an accelerated blood clearance and a marked reduction in bioavailability [63-65]. The anti-PEG immune response involves B cells from the marginal zone of the spleen and is characterized by a lack of immunoglobulin class switching as well as the absence of generation of memory B cells [66-70]. Therefore, the immune response 
develops in a thymo-independent manner. As PEG is constituted of multiple repeated patterns, it may be regarded as a type II antigen, similar to bacterial polysaccharides, and it may simultaneously engage several BcR expressed on the surface of B cells. This massive cross-linking of BcR is sufficient to induce the full differentiation of naïve B cells into IgMsecreting plasmocytes. Lastly, some reports suggest that PEGylated biotherapeutics are able to activate the complement system [71,72]. Complement activation leads to opsonization of the biotherapeutics by $\mathrm{C} 3$-derived fragments that may promote interactions with macrophages through complement receptors. This may participate in the accelerated decay of PEGylated pharmaceuticals, but may also induce pro-inflammatory anaphylatoxins, that can activate granulocytes and trigger hypersensitivity responses. Such a phenomenon probably accounts for the few cases of anaphylaxis observed in clinical trials, for instance in the case of treatment with PEGylated liposomal doxorubicin [73].

Of note, immunogenicity studies in mice have shown that some forms of PEGylated FVIII evoke an anti-FVIII immune response, and are able to break immune tolerance the FVIII moiety [74]. However the incriminated molecule exhibited 12 molecules of PEG per molecule of FVIII and drastically reduced specific activity, thus highlighting probable major structural modifications.

\section{Conclusions}

The development of a new generation of therapeutic coagulation factors is revolutionizing the treatment of patients with hemophilia. Specifically, novel products with extended half-lives should facilitate patient management, resulting in a drastically improved quality of life. Indeed, the reduced frequency of drug administration and the prolonged circulation time of the hemostatic drugs should provide a longer protection of the patients from micro-bleeds in joints, thus minimizing co-morbidity. In the particular case of pro-coagulant FVIII, among the 
three strategies developed to date, only Fc-fusion and PEGylation have yielded encouraging results and been translated to the patients, while albumin-fusion FVIII has failed. Yet, several issues remain to be addressed to develop products with unquestioned efficacy and safety.

One such question addresses the somewhat limited gain in half-lives of the Fc-fused and PEGylated FVIII products that do not exceed the circulation time of FVIII imposed by binding to its chaperone VWF. It is probable that VWF is endowed with a double-edged role towards FVIII catabolism, on one hand reducing the binding of FVIII to major catabolic receptors, but also on the other hand participating in, and probably leading, FVIII endocytosis by macrophages both in vivo and in vitro $[75,76]$. Under such conditions, it is probable that drastic gains in FVIII half-life for the new generation therapeutics will be achieved in setups that eliminate the part of FVIII catabolism mediated by VWF moieties, as may be the case in a recently developed recombinant FVIII-Fc/D'D3-Fc construct (patent WO 2013106787 A1). One other important issue is that of the ever-astonishing intrinsic immunogenicity of the very FVIII molecule. While most biotherapeutics induce anti-drug antibodies in $<5 \%$ of the patients, FVIII triggers inhibitory immune responses in up to $30 \%$ of patients with hemophilia A. FVIII remains an enigma in terms of immunogenicity, in view of the facts that it is injected intravenously, a known tolerogenic route of administration and in low amounts. A role for its pro-coagulant function in generating a burst of thrombin [77,78], the presence of incomplete glycans on its heavy and light chain that may favor immunogenic uptake by antigenpresenting cells $[79,80]$ as well as the presence of particularly immune-dominant $\mathrm{T}$ cell epitopes have been proposed to explain anti-FVIII immune responses [81-83]. FVIII immunogenicity is further highlighted by its capacity to confer immunogenicity to otherwise immunologically inert molecules. This has been demonstrated by the work of van Helden et al in the case of PEG [74], and may also be observed in the case of fused protein moieties as seen in our unpublished experiments wherein fusion of the FVIII light chain to hemagglutinin 
drastically increased the anti-hemagglutinin immune response in FVIII-deficient mice (Figure 2).

Another critical issue is the mechanistic investigation of the immunogenicity of newly generated coagulation factors and the requirement of experimental models that mimic immune responses observed in humans and that are devoid of biasing inter-species immune reactions. In particular, studying the immunogenicity of Fc-fused FVIII in FVIII-deficient mice may be biased by putative immune responses directed to the human Fc fragment, which would presumably not occur in the patients. Ideally, the immunogenicity of human FVIII should be evaluation using appropriate humanized animal models able to mount IgGmediated humoral immune responses, in order to obtain relevant and basic immunological insights translatable to the bedside. Resolving the aforementioned concerns, the in-depth immunological investigations of new generation long-lasting coagulation factors should be of priority in the hemophilia research domain. 


\section{Acknowledgments}

This work was supported by Institut national de la santé et de la recherche médicale (INSERM), Centre National de la Recherche Scientifique (CNRS), Université Pierre et Marie Curie (UPMC) Paris 6, and the Innovative Medicines Initiative Joint Undertaking under grant agreement n. 115303, with resources from the European Union's Seventh Framework Programme (FP7/2007-2013) and EFPIA companies' in kind contribution. Mathieu Ing is the recipient of a fellowship from Ministère de l'enseignement supérieur et de la recherche (France).

The authors declare no conflict of interest. 


\section{Figures}

Figure 1. Potential effects of FVIII alterations on the onset of the immune response against FVIII. Panel A. The anti-FVIII immune response is considered as a classical Tdependent immune response where exogenous FVIII is endocytosed by antigen presenting cells (APC), processed and presented to naïve FVIII-specific $\mathrm{T}$ lymphocytes that get activated. The latter will activate B lymphocytes, which will differentiate into inhibitory antibody-secreting plasma cells. Panel B. Fusion of Fc fragment to FVIII may facilitate the cross-linking of surface B cell receptors $(\mathrm{BcR})$ and inhibitory FcyRIIb receptors, thus anergizing B cells (left). Fc fragment may interact with Fc $\gamma$ RIIb receptors expressed on APC and impede their maturation, leading to FVIII-derived peptides antigenic presentation in a tolerogenic context (middle). Fc moiety might bring about regulatory $\mathrm{T}$ cell epitopes (Tregitopes) that would boost Fc-specific regulatory $\mathrm{T}$ cells (Tregs). Tregs would then induce a bystander tolerance to FVIII (right). Panel C. PEGylation of FVIII may prevent the uptake of FVIII by APC. Even if it is endocytosed, PEG moiety may alter FVIII processing and presentation to $\mathrm{T}$ lymphocytes. Finally, PEGylation may also mask B cells epitopes and prevent FVIII recognition by neutralizing antibodies.

Figure 2. Adjuvant effect of the light chain of human FVIII. The recombinant first domain of hemagglutinin (HA1), and the recombinant fused A3C1C2-HA1 (LCh-HA1) were produced using the HKB11 eukaryotic cell line. The light chain of FVIII (a3A3C1C2, LCh) was purified from therapeutic FVIII. FVIII-deficient mice were injected with the different proteins ( 1 pmol of each protein/mouse) once a week for four weeks. Five days after the fourth injection, anti-HA1 IgG were detected by ELISA. Levels of anti-HA1 IgG are depicted in arbitrary unit based on the optical density measured at $492 \mathrm{~nm}$, and were statistically compared using the two-sided non-parametric Mann-Whitney test. Individual symbols 
represent levels of specific $\operatorname{IgG}$ for each individual mouse, and horizontal bars represent mean \pm SEM. 


\section{References}

[1] D.C. Roopenian, S. Akilesh, FcRn: the neonatal Fc receptor comes of age, Nat. Rev. Immunol. 7 (2007) 715-725. doi:10.1038/nri2155.

[2] J.A. Dumont, T. Liu, S.C. Low, X. Zhang, G. Kamphaus, P. Sakorafas, et al., Prolonged activity of a recombinant factor VIII-Fc fusion protein in hemophilia A mice and dogs, Blood. 119 (2012) 3024-3030. doi:10.1182/blood-2011-08-367813.

[3] J.S. Powell, N.C. Josephson, D. Quon, M.V. Ragni, G. Cheng, E. Li, et al., Safety and prolonged activity of recombinant factor VIII Fc fusion protein in hemophilia A patients, Blood. 119 (2012) 3031-3037. doi:10.1182/blood-2011-09-382846.

[4] A.D. Shapiro, M.V. Ragni, R. Kulkarni, J. Oldenberg, A. Srivastava, D.V. Quon, et al., Recombinant factor VIII Fc fusion protein: extended-interval dosing maintains low bleeding rates and correlates with von Willebrand factor levels, J. Thromb. Haemost. JTH. 12 (2014) 1788-1800. doi:10.1111/jth.12723.

[5] J. Mahlangu, J.S. Powell, M.V. Ragni, P. Chowdary, N.C. Josephson, I. Pabinger, et al., Phase 3 study of recombinant factor VIII Fc fusion protein in severe hemophilia A, Blood. 123 (2014) 317-325. doi:10.1182/blood-2013-10-529974.

[6] G. Young, J. Mahlangu, R. Kulkarni, B. Nolan, R. Liesner, J. Pasi, et al., Recombinant factor VIII Fc fusion protein for the prevention and treatment of bleeding in children with severe hemophilia A, J. Thromb. Haemost. JTH. 13 (2015) 967-977. doi: $10.1111 /$ jth. 12911 .

[7] S.C. Gouw, J.G. van der Bom, H. Marijke van den Berg, Treatment-related risk factors of inhibitor development in previously untreated patients with hemophilia A: the CANAL cohort study, Blood. 109 (2007) 4648-4654. doi:10.1182/blood-2006-11056291.

[8] F.A. Harding, M.M. Stickler, J. Razo, R.B. DuBridge, The immunogenicity of humanized and fully human antibodies, mAbs. 2 (2010) 256-265.

[9] P.M. Guyre, R.F. Graziano, J. Goldstein, P.K. Wallace, P.M. Morganelli, K. Wardwell, et al., Increased potency of Fc-receptor-targeted antigens, Cancer Immunol. Immunother. CII. 45 (1997) 146-148.

[10] K. Konduru, S.B. Bradfute, J. Jacques, M. Manangeeswaran, S. Nakamura, S. Morshed, et al., Ebola virus glycoprotein Fc fusion protein confers protection against lethal challenge in vaccinated mice, Vaccine. 29 (2011) 2968-2977. doi:10.1016/j.vaccine.2011.01.113.

[11] A. Pincetic, S. Bournazos, D.J. DiLillo, J. Maamary, T.T. Wang, R. Dahan, et al., Type I and type II Fc receptors regulate innate and adaptive immunity, Nat. Immunol. 15 (2014) 707-716. doi:10.1038/ni.2939.

[12] S. Krishnamoorthy, T. Liu, D. Drager, S. Patarroyo-White, E.S. Chhabra, S. Peters, et al., Recombinant Factor VIII Fc (rFVIIIFc) Fusion Protein Reduces Immunogenicity and Induces Tolerance in Hemophilia A Mice, Cell. Immunol. In press (2015).

[13] A. Navarrete, S. Dasgupta, S. Delignat, G. Caligiuri, O.D. Christophe, J. Bayry, et al., Splenic marginal zone antigen-presenting cells are critical for the primary allo-immune response to therapeutic factor VIII in hemophilia A, J. Thromb. Haemost. JTH. 7 (2009) 1816-1823. doi:10.1111/j.1538-7836.2009.03571.x.

[14] A. van der Flier, Z. Liu, S. Tan, K. Chen, D. Drager, T. Liu, et al., FcRn Rescues Recombinant Factor VIII Fc Fusion Protein from a VWF Independent FVIII Clearance Pathway in Mouse Hepatocytes, PloS One. 10 (2015) e0124930. doi:10.1371/journal.pone.0124930.

[15] P. Bruhns, Properties of mouse and human IgG receptors and their contribution to disease models, Blood. 119 (2012) 5640-5649. doi:10.1182/blood-2012-01-380121. 
[16] F. Nimmerjahn, J.V. Ravetch, Fcgamma receptors: old friends and new family members, Immunity. 24 (2006) 19-28. doi:10.1016/j.immuni.2005.11.010.

[17] D.D. Desai, S.O. Harbers, M. Flores, L. Colonna, M.P. Downie, A. Bergtold, et al., Fc gamma receptor IIB on dendritic cells enforces peripheral tolerance by inhibiting effector T cell responses, J. Immunol. Baltim. Md 1950. 178 (2007) 6217-6226.

[18] H.M. Horton, S.Y. Chu, E.C. Ortiz, E. Pong, S. Cemerski, I.W.L. Leung, et al., Antibody-mediated coengagement of Fc $\gamma$ RIIb and B cell receptor complex suppresses humoral immunity in systemic lupus erythematosus, J. Immunol. Baltim. Md 1950. 186 (2011) 4223-4233. doi:10.4049/jimmunol.1003412.

[19] S. Werwitzke, N. Vollack, M. von Hornung, K. Kalippke, J. Kutzschbach, A. Trummer, et al., Deletion or inhibition of Fc gamma receptor 2B (CD32) prevents FVIII-specific activation of memory B cells in vitro, Thromb. Haemost. 114 (2015). doi:10.1160/TH14-06-0535.

[20] A.S. De Groot, L. Cousens, F. Mingozzi, W. Martin, Tregitope peptides: the active pharmaceutical ingredient of IVIG?, Clin. Dev. Immunol. 2013 (2013) 493138. doi: $10.1155 / 2013 / 493138$.

[21] D.J. Hui, E. Basner-Tschakarjan, Y. Chen, R.J. Davidson, G. Buchlis, M. Yazicioglu, et al., Modulation of CD8+ T cell responses to AAV vectors with IgG-derived MHC class II epitopes, Mol. Ther. J. Am. Soc. Gene Ther. 21 (2013) 1727-1737. doi:10.1038/mt.2013.166.

[22] W.R. Heath, F.R. Carbone, The skin-resident and migratory immune system in steady state and memory: innate lymphocytes, dendritic cells and T cells, Nat. Immunol. 14 (2013) 978-985. doi:10.1038/ni.2680.

[23] E. Shklovskaya, B.J. O’Sullivan, L.G. Ng, B. Roediger, R. Thomas, W. Weninger, et al., Langerhans cells are precommitted to immune tolerance induction, Proc. Natl. Acad. Sci. U. S. A. 108 (2011) 18049-18054. doi:10.1073/pnas.1110076108.

[24] J.H. Grubb, C. Vogler, Y. Tan, G.N. Shah, A.F. MacRae, W.S. Sly, Infused Fc-tagged beta-glucuronidase crosses the placenta and produces clearance of storage in utero in mucopolysaccharidosis VII mice, Proc. Natl. Acad. Sci. U. S. A. 105 (2008) 83758380. doi:10.1073/pnas.0803715105.

[25] N. Gupta, S. Culina, Y. Meslier, J. Dimitrov, C. Arnoult, S. Delignat, et al., Regulation of immune responses to protein therapeutics by transplacental induction of T cell tolerance, Sci. Transl. Med. 7 (2015) 275ra21. doi:10.1126/scitranslmed.aaa1957.

[26] D. Sleep, J. Cameron, L.R. Evans, Albumin as a versatile platform for drug half-life extension, Biochim. Biophys. Acta. 1830 (2013) 5526-5534. doi:10.1016/j.bbagen.2013.04.023.

[27] M. Subramanyam, Immunogenicity of Biotherapeutics-An Overview, J. Immunotoxicol. 3 (2006) 151-156. doi:10.1080/15476910600845740.

[28] J.J. Neumiller, Clinical pharmacology of incretin therapies for type 2 diabetes mellitus: implications for treatment, Clin. Ther. 33 (2011) 528-576. doi:10.1016/j.clinthera.2011.04.024.

[29] R.E. Pratley, M.A. Nauck, A.H. Barnett, M.N. Feinglos, F. Ovalle, I. Harman-Boehm, et al., Once-weekly albiglutide versus once-daily liraglutide in patients with type 2 diabetes inadequately controlled on oral drugs (HARMONY 7): a randomised, openlabel, multicentre, non-inferiority phase 3 study, Lancet Diabetes Endocrinol. 2 (2014) 289-297. doi:10.1016/S2213-8587(13)70214-6.

[30] S. Schulte, Innovative coagulation factors: albumin fusion technology and recombinant single-chain factor VIII, Thromb. Res. 131 Suppl 2 (2013) S2-6. doi:10.1016/S00493848(13)70150-6. 
[31] T. Weimer, W. Wormsbächer, U. Kronthaler, W. Lang, U. Liebing, S. Schulte, Prolonged in-vivo half-life of factor VIIa by fusion to albumin, Thromb. Haemost. 99 (2008) 659-667. doi:10.1160/TH07-08-0525.

[32] G. Golor, D. Bensen-Kennedy, S. Haffner, R. Easton, K. Jung, T. Moises, et al., Safety and pharmacokinetics of a recombinant fusion protein linking coagulation factor VIIa with albumin in healthy volunteers, J. Thromb. Haemost. JTH. 11 (2013) 1977-1985. doi:10.1111/jth.12409.

[33] U. Martinowitz, T. Lissitchkov, A. Lubetsky, G. Jotov, T. Barazani-Brutman, C. Voigt, et al., Results of a phase I/II open-label, safety and efficacy trial of coagulation factor IX (recombinant), albumin fusion protein in haemophilia B patients, Haemoph. Off. J. World Fed. Hemoph. (2015). doi:10.1111/hae.12721.

[34] J.S. Powell, Novel Approaches for Hemophilia Treatment, Medscape. (2015). http://www.medscape.com/viewarticle/841623 (accessed October 22, 2015).

[35] S.I. Lim, Y.S. Hahn, I. Kwon, Site-specific albumination of a therapeutic protein with multi-subunit to prolong activity in vivo, J. Control. Release Off. J. Control. Release Soc. 207 (2015) 93-100. doi:10.1016/j.jconrel.2015.04.004.

[36] P. Caraceni, M. Tufoni, M.E. Bonavita, Clinical use of albumin, Blood Transfus. Trasfus. Sangue. 11 Suppl 4 (2013) s18-25. doi:10.2450/2013.005s.

[37] C.A. Weber, P.J. Mehta, M. Ardito, L. Moise, B. Martin, A.S. De Groot, T cell epitope: friend or foe? Immunogenicity of biologics in context, Adv. Drug Deliv. Rev. 61 (2009) 965-976. doi:10.1016/j.addr.2009.07.001.

[38] D. Kolbus, M. Wigren, I. Ljungcrantz, I. Söderberg, R. Alm, H. Björkbacka, et al., Immunization with cationized BSA inhibits progression of disease in ApoBec-1/LDL receptor deficient mice with manifest atherosclerosis, Immunobiology. 216 (2011) 663669. doi:10.1016/j.imbio.2010.11.003.

[39] M.G. Saifer, R. Somack, L.D. Williams, Plasma clearance and immunologic properties of long-acting superoxide dismutase prepared using 35,000 to 120,000 dalton polyethylene glycol, Adv. Exp. Med. Biol. 366 (1994) 377-387.

[40] F.M. Veronese, Peptide and protein PEGylation: a review of problems and solutions, Biomaterials. 22 (2001) 405-417.

[41] M. Hamidi, A. Azadi, P. Rafiei, Pharmacokinetic consequences of pegylation, Drug Deliv. 13 (2006) 399-409. doi:10.1080/10717540600814402.

[42] B. Mei, C. Pan, H. Jiang, H. Tjandra, J. Strauss, Y. Chen, et al., Rational design of a fully active, long-acting PEGylated factor VIII for hemophilia A treatment, Blood. 116 (2010) 270-279. doi:10.1182/blood-2009-11-254755.

[43] T.E. Coyle, M.T. Reding, J.C. Lin, L.A. Michaels, A. Shah, J. Powell, Phase I study of BAY 94-9027, a PEGylated B-domain-deleted recombinant factor VIII with an extended half-life, in subjects with hemophilia A, J. Thromb. Haemost. JTH. 12 (2014) 488-496.

[44] P.L. Turecek, M.J. Bossard, M. Graninger, H. Gritsch, W. Höllriegl, M. Kaliwoda, et al., BAX 855, a PEGylated rFVIII product with prolonged half-life. Development, functional and structural characterisation, Hämostaseologie. 32 Suppl 1 (2012) S29-38.

[45] B.A. Konkle, O. Stasyshyn, P. Chowdary, D.H. Bevan, T. Mant, M. Shima, et al., Pegylated, full-length, recombinant factor VIII for prophylactic and on-demand treatment of severe hemophilia A, Blood. 126 (2015) 1078-1085. doi:10.1182/blood2015-03-630897.

[46] H.R. Stennicke, M. Kjalke, D.M. Karpf, K.W. Balling, P.B. Johansen, T. Elm, et al., A novel B-domain O-glycoPEGylated FVIII (N8-GP) demonstrates full efficacy and prolonged effect in hemophilic mice models, Blood. 121 (2013) 2108-2116. doi:10.1182/blood-2012-01-407494. 
[47] A.E. Pastoft, M. Ezban, M. Tranholm, J. Lykkesfeldt, B. Lauritzen, Prolonged effect of a new O-glycoPEGylated FVIII (N8-GP) in a murine saphenous vein bleeding model, Haemoph. Off. J. World Fed. Hemoph. 19 (2013) 913-919. doi:10.1111/hae.12198.

[48] A. Tiede, B. Brand, R. Fischer, K. Kavakli, S.R. Lentz, T. Matsushita, et al., Enhancing the pharmacokinetic properties of recombinant factor VIII: first-in-human trial of glycoPEGylated recombinant factor VIII in patients with hemophilia A, J. Thromb. Haemost. JTH. 11 (2013) 670-678. doi:10.1111/jth.12161.

[49] A. Abuchowski, J.R. McCoy, N.C. Palczuk, T. van Es, F.F. Davis, Effect of covalent attachment of polyethylene glycol on immunogenicity and circulating life of bovine liver catalase, J. Biol. Chem. 252 (1977) 3582-3586.

[50] J.X. P. PAZ, PEGylated FVIII Exhibits Reduced Immunogenicity in Hemophilia A Mice and In Vitro in Human Cells, Haemophilia. 18 (2012).

[51] R. Webster, E. Didier, P. Harris, N. Siegel, J. Stadler, L. Tilbury, et al., PEGylated proteins: evaluation of their safety in the absence of definitive metabolism studies, Drug Metab. Dispos. Biol. Fate Chem. 35 (2007) 9-16. doi:10.1124/dmd.106.012419.

[52] A. Bendele, J. Seely, C. Richey, G. Sennello, G. Shopp, Short Communication: Renal Tubular Vacuolation in Animals Treated with Polyethylene-Glycol-Conjugated Proteins, Toxicol. Sci. 42 (1998) 152-157. doi:10.1093/toxsci/42.2.152.

[53] D.G. Rudmann, J.T. Alston, J.C. Hanson, S. Heidel, High Molecular Weight Polyethylene Glycol Cellular Distribution and PEG-associated Cytoplasmic Vacuolation Is Molecular Weight Dependent and Does Not Require Conjugation to Proteins, Toxicol. Pathol. 41 (2013) 970-983. doi:10.1177/0192623312474726.

[54] A. Baumann, D. Tuerck, S. Prabhu, L. Dickmann, J. Sims, Pharmacokinetics, metabolism and distribution of PEGs and PEGylated proteins: quo vadis?, Drug Discov. Today. 19 (2014) 1623-1631. doi:10.1016/j.drudis.2014.06.002.

[55] A.T. Viau, A. Abuchowski, S. Greenspan, F.F. Davis, Safety evaluation of free radical scavengers PEG-catalase and PEG-superoxide dismutase, J. Free Radic. Biol. Med. 2 (1986) 283-288.

[56] M.A. Young, A. Malavalli, N. Winslow, K.D. Vandegriff, R.M. Winslow, Toxicity and hemodynamic effects after single dose administration of MalPEG-hemoglobin (MP4) in rhesus monkeys, Transl. Res. J. Lab. Clin. Med. 149 (2007) 333-342. doi:10.1016/j.trsl.2006.09.007.

[57] R. Stidl, S. Fuchs, M. Bossard, J. Siekmann, P.L. Turecek, M. Putz, Safety of PEGylated recombinant human full-length coagulation factor VIII (BAX 855) in the overall context of PEG and PEG conjugates, Haemoph. Off. J. World Fed. Hemoph. (2015). doi:10.1111/hae.12762.

[58] R.P. Garay, R. El-Gewely, J.K. Armstrong, G. Garratty, P. Richette, Antibodies against polyethylene glycol in healthy subjects and in patients treated with PEG-conjugated agents, Expert Opin. Drug Deliv. 9 (2012) 1319-1323. doi:10.1517/17425247.2012.720969.

[59] A.W. Richter, E. Akerblom, Antibodies against polyethylene glycol produced in animals by immunization with monomethoxy polyethylene glycol modified proteins, Int. Arch. Allergy Appl. Immunol. 70 (1983) 124-131.

[60] Y. Mima, Y. Hashimoto, T. Shimizu, H. Kiwada, T. Ishida, Anti-PEG IgM Is a Major Contributor to the Accelerated Blood Clearance of Polyethylene Glycol-Conjugated Protein, Mol. Pharm. 12 (2015) 2429-2435. doi:10.1021/acs.molpharmaceut.5b00144.

[61] H. Tillmann, N.J. Ganson, K. Patel, A.J. Thompson, M. Abdelmalek, T. Moody, et al., High prevalence of pre-existing antibodies against polyethylene glycol (PEG) in hepatitis $\mathrm{C}(\mathrm{HCV})$ patients which is not associated with impaired response to PEG- 
interferon, J. Hepatol. 52, Supplement 1 (2010) S129. doi:10.1016/S01688278(10)60309-1.

[62] J.K. Armstrong, G. Hempel, S. Koling, L.S. Chan, T. Fisher, H.J. Meiselman, et al., Antibody against poly(ethylene glycol) adversely affects PEG-asparaginase therapy in acute lymphoblastic leukemia patients, Cancer. 110 (2007) 103-111. doi:10.1002/cncr.22739.

[63] T. Shimizu, Y. Mima, Y. Hashimoto, M. Ukawa, H. Ando, H. Kiwada, et al., Anti-PEG IgM and complement system are required for the association of second doses of PEGylated liposomes with splenic marginal zone B cells, Immunobiology. 220 (2015) 1151-1160. doi:10.1016/j.imbio.2015.06.005.

[64] T. Ishida, M. Harada, X.Y. Wang, M. Ichihara, K. Irimura, H. Kiwada, Accelerated blood clearance of PEGylated liposomes following preceding liposome injection: effects of lipid dose and PEG surface-density and chain length of the first-dose liposomes, J. Control. Release Off. J. Control. Release Soc. 105 (2005) 305-317. doi:10.1016/j.jconrel.2005.04.003.

[65] A.S. Abu Lila, H. Kiwada, T. Ishida, The accelerated blood clearance (ABC) phenomenon: clinical challenge and approaches to manage, J. Control. Release Off. J. Control. Release Soc. 172 (2013) 38-47. doi:10.1016/j.jconrel.2013.07.026.

[66] T. Ishida, M. Ichihara, X. Wang, H. Kiwada, Spleen plays an important role in the induction of accelerated blood clearance of PEGylated liposomes, J. Control. Release Off. J. Control. Release Soc. 115 (2006) 243-250. doi:10.1016/j.jconrel.2006.08.001.

[67] A.S. Abu Lila, M. Ichihara, T. Shimizu, T. Ishida, H. Kiwada, Ex-vivo/in-vitro antipolyethylene glycol (PEG) immunoglobulin M production from murine splenic B cells stimulated by PEGylated liposome, Biol. Pharm. Bull. 36 (2013) 1842-1848.

[68] M. Ichihara, T. Shimizu, A. Imoto, Y. Hashiguchi, Y. Uehara, T. Ishida, et al., AntiPEG IgM Response against PEGylated Liposomes in Mice and Rats, Pharmaceutics. 3 (2010) 1-11. doi:10.3390/pharmaceutics3010001.

[69] H. Koide, T. Asai, K. Hatanaka, S. Akai, T. Ishii, E. Kenjo, et al., T cell-independent B cell response is responsible for $\mathrm{ABC}$ phenomenon induced by repeated injection of PEGylated liposomes, Int. J. Pharm. 392 (2010) 218-223. doi:10.1016/j.ijpharm.2010.03.022.

[70] Y. Hashimoto, T. Shimizu, A.S. Abu Lila, T. Ishida, H. Kiwada, Relationship between the concentration of anti-polyethylene glycol (PEG) immunoglobulin $\mathrm{M}(\mathrm{IgM})$ and the intensity of the accelerated blood clearance $(\mathrm{ABC})$ phenomenon against PEGylated liposomes in mice, Biol. Pharm. Bull. 38 (2015) 417-424. doi:10.1248/bpb.b14-00653.

[71] I. Hamad, A.C. Hunter, J. Szebeni, S.M. Moghimi, Poly(ethylene glycol)s generate complement activation products in human serum through increased alternative pathway turnover and a MASP-2-dependent process, Mol. Immunol. 46 (2008) 225-232. doi:10.1016/j.molimm.2008.08.276.

[72] J. Szebeni, F. Muggia, A. Gabizon, Y. Barenholz, Activation of complement by therapeutic liposomes and other lipid excipient-based therapeutic products: prediction and prevention, Adv. Drug Deliv. Rev. 63 (2011) 1020-1030. doi:10.1016/j.addr.2011.06.017.

[73] A. Chanan-Khan, J. Szebeni, S. Savay, L. Liebes, N.M. Rafique, C.R. Alving, et al., Complement activation following first exposure to pegylated liposomal doxorubicin (Doxil): possible role in hypersensitivity reactions, Ann. Oncol. Off. J. Eur. Soc. Med. Oncol. ESMO. 14 (2003) 1430-1437.

[74] P.M. van Helden, S. Unterthurner, C. Hermann, M. Schuster, R.U. Ahmad, A.N. Schiviz, et al., Maintenance and break of immune tolerance against human factor VIII 
in a new transgenic hemophilic mouse model, Blood. 118 (2011) 3698-3707. doi:10.1182/blood-2010-11-316521.

[75] L. Castro-Núñez, I. Dienava-Verdoold, E. Herczenik, K. Mertens, A.B. Meijer, Shear stress is required for the endocytic uptake of the factor VIII-von Willebrand factor complex by macrophages, J. Thromb. Haemost. JTH. 10 (2012) 1929-1937. doi:10.1111/j.1538-7836.2012.04860.x.

[76] C.J. van Schooten, S. Shahbazi, E. Groot, B.D. Oortwijn, H.M. van den Berg, C.V. Denis, et al., Macrophages contribute to the cellular uptake of von Willebrand factor and factor VIII in vivo, Blood. 112 (2008) 1704-1712. doi:10.1182/blood-2008-01133181.

[77] J. Skupsky, A.-H. Zhang, Y. Su, D.W. Scott, A role for thrombin in the initiation of the immune response to therapeutic factor VIII, Blood. 114 (2009) 4741-4748. doi:10.1182/blood-2008-10-186452.

[78] B. Gangadharan, S. Delignat, V. Ollivier, N. Gupta, N. Mackman, S.V. Kaveri, et al., Role of coagulation-associated processes on factor VIII immunogenicity in a mouse model of severe hemophilia A, J. Thromb. Haemost. JTH. 12 (2014) 2065-2069. doi: $10.1111 /$ jth. 12740 .

[79] S. Dasgupta, A.-M. Navarrete, J. Bayry, S. Delignat, B. Wootla, S. André, et al., A role for exposed mannosylations in presentation of human therapeutic self-proteins to CD4+ T lymphocytes, Proc. Natl. Acad. Sci. U. S. A. 104 (2007) 8965-8970. doi:10.1073/pnas.0702120104.

[80] Y. Repessé, S. Dasgupta, A.-M. Navarrete, S. Delignat, S.V. Kaveri, S. LacroixDesmazes, Mannose-sensitive receptors mediate the uptake of factor VIII therapeutics by human dendritic cells, J. Allergy Clin. Immunol. 129 (2012) 1172-1173; author reply 1174-1175. doi:10.1016/j.jaci.2012.01.048.

[81] C. Yanover, N. Jain, G. Pierce, T.E. Howard, Z.E. Sauna, Pharmacogenetics and the immunogenicity of protein therapeutics, Nat. Biotechnol. 29 (2011) 870-873. doi:10.1038/nbt.2002.

[82] A.D. Pashov, T. Calvez, L. Gilardin, B. Maillère, Y. Repessé, J. Oldenburg, et al., In silico calculated affinity of FVIII-derived peptides for HLA class II alleles predicts inhibitor development in haemophilia A patients with missense mutations in the F8 gene, Haemoph. Off. J. World Fed. Hemoph. 20 (2014) 176-184. doi:10.1111/hae.12276.

[83] A.J. Shepherd, S. Skelton, C.E. Sansom, K. Gomez, D.S. Moss, D.P. Hart, A large-scale computational study of inhibitor risk in non-severe haemophilia A, Br. J. Haematol. 168 (2015) 413-420. doi:10.1111/bjh.13131. 


\section{Figure 1}

\section{A - Anti-FVIII immune response}

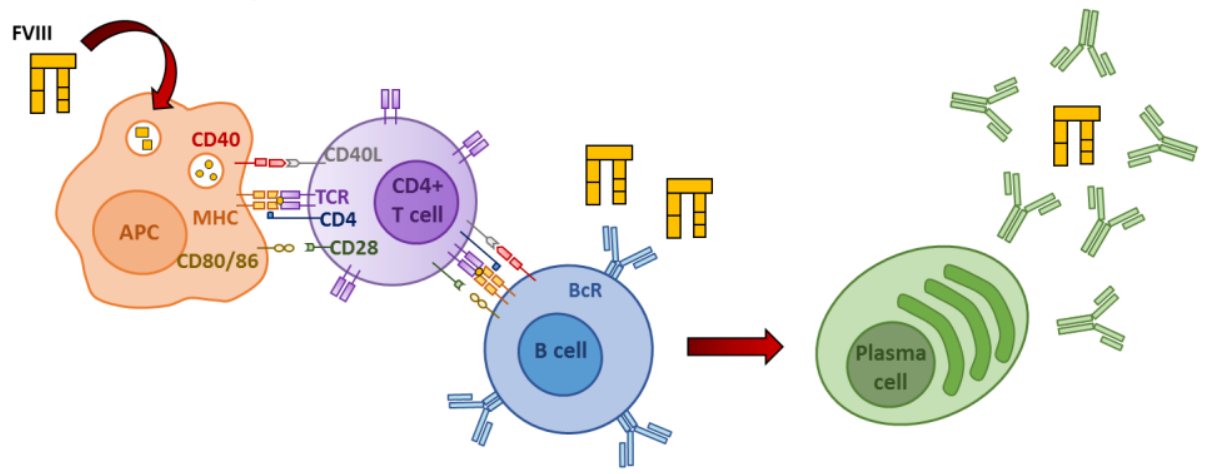

B - Fc-fused FVIII

B CELL ANERGY
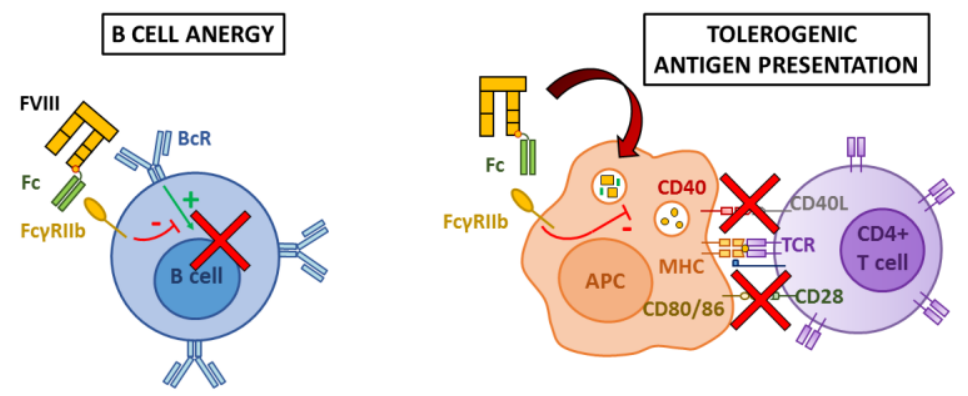

- APC maturation blockade

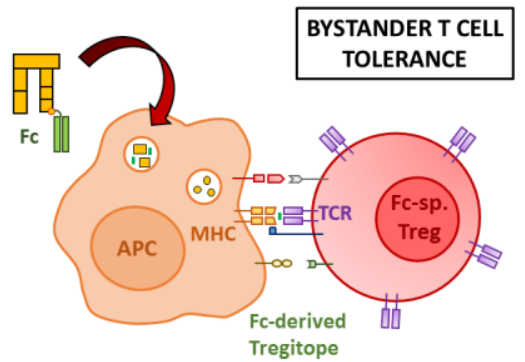

- Inhibition of B cells activation

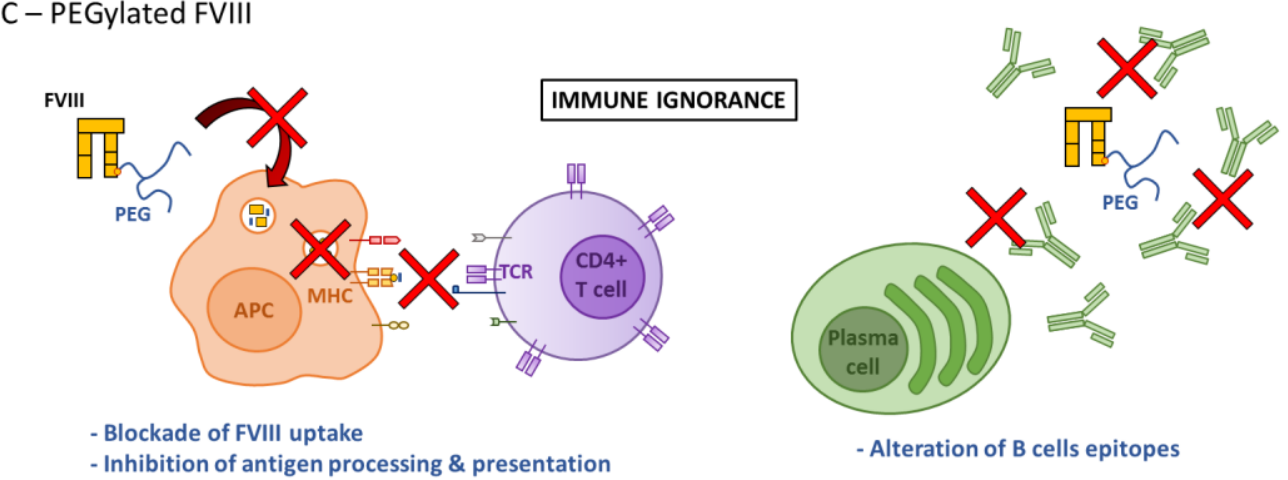


Figure 2

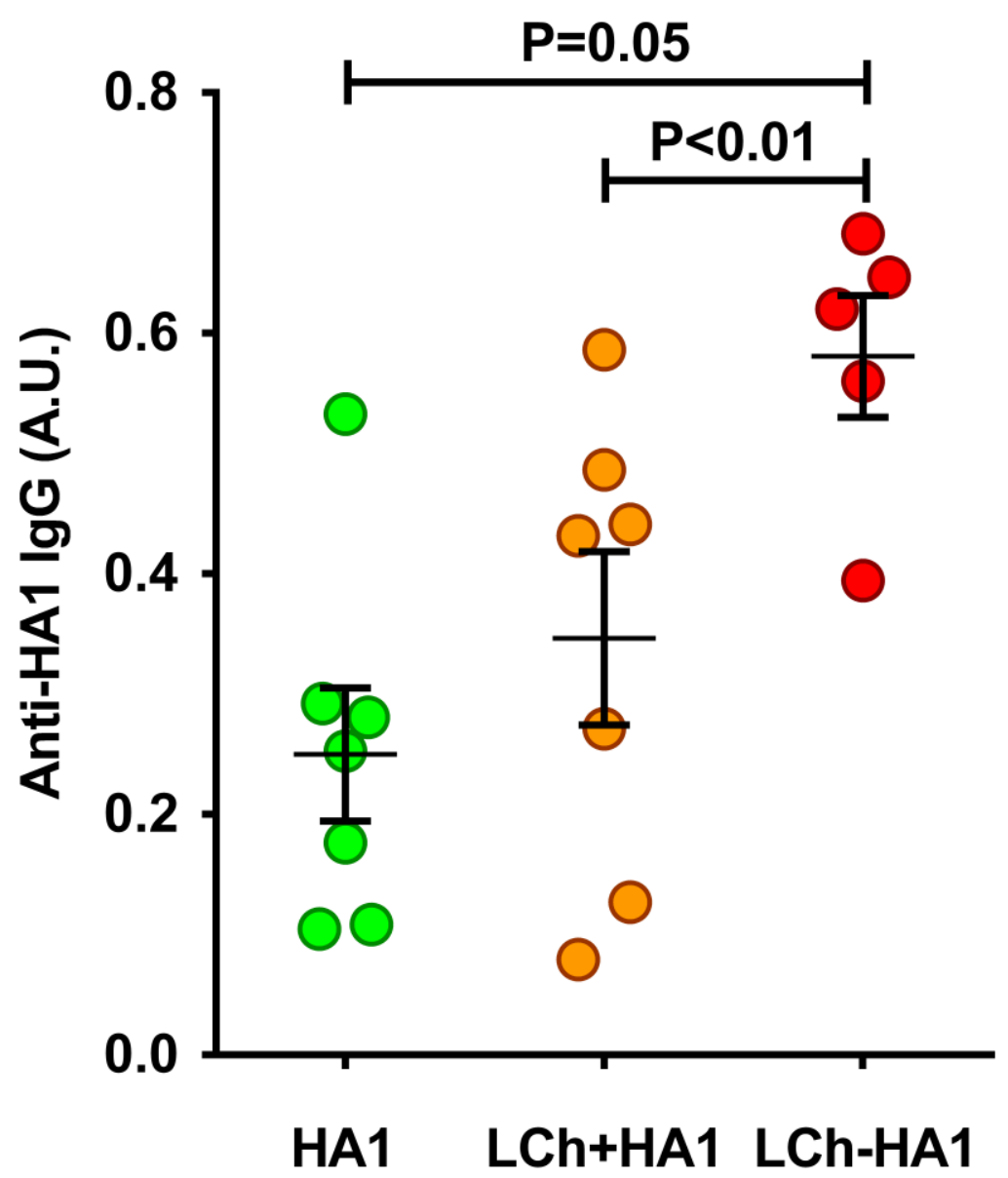

